\title{
Phase I Clinical Trials in 85 Patients with Gynecologic Cancer: The M. D. Anderson Cancer Center Experience
}

\author{
John Moroney ${ }^{1}$, Jennifer Wheler ${ }^{2}$, David Hong ${ }^{2}$, Aung Naing ${ }^{2}$, Gerald Falchook ${ }^{2}$, Diane \\ Bodurka $^{1}$, Robert Coleman ${ }^{1}$, Karen Lu ${ }^{1}$, Lianchun Xiao ${ }^{3}$, and Razelle Kurzrock ${ }^{2}$ \\ ${ }^{1}$ Department of Gynecologic Oncology, The University of Texas M.D. Anderson Cancer Center, \\ Houston, Texas \\ 2 Phase I Clinical Trials Program, Department of Investigational Cancer Therapeutics; The \\ University of Texas M. D. Anderson Cancer Center, Houston, Texas \\ ${ }^{3}$ Department of Biostatistics, The University of Texas M. D. Anderson Cancer Center, Houston, \\ Texas
}

\begin{abstract}
Objective-Disseminated gynecologic cancers are usually fatal due to chemoresistance. Recently, rationally-developed, targeted agents are entering the early clinical trials setting. We assessed patients with metastatic gynecologic cancers in a dedicated Phase I clinical trials clinic in order to determine their outcome.
\end{abstract}

Methods-We reviewed records for 89 consecutive patients with gynecologic cancers referred to the Phase I Clinical Trials Program, 85 (96\%) of whom were treated on $\geq 1$ trial.

Results-Cancer diagnoses were: ovarian $(\mathrm{N}=43)$, uterine $(\mathrm{N}=19)$, cervix $(\mathrm{N}=17)$, and other. Median age was 58 years; median number of prior cytotoxic regimens, five. Two patients (2.4\%) achieved a CR, four (4.7\%), a PR, and eight (9.4\%), SD $\geq$ six months (total CR/PR/SD $\geq$ six months $=16.5 \%)$ for the first phase I trial. Twenty-five patients enrolled on a second trial and three on a third $(\mathrm{N}=113$ trials total). Combining response data for all trials, of the 85 patients, two achieved $\mathrm{CR}$ (2.4\%), nine achieved PR (10.6\%), and $12(14 \%)$ had SD for $\geq$ six months. One-year survival was $30 \%$ (95\% C.I., $21 \%$ to $44 \%$ ). There was no difference in time-to-treatment failure (TTF) on Phase I versus the patient's last standard treatment.

Conclusion-Twenty-three of 85 patients (27\%) with advanced, heavily-pretreated, gynecologic cancers achieved $\mathrm{CR} / \mathrm{PR} / \mathrm{SD} \geq$ six months on a phase I trial, and overall TTF on phase I was comparable to that of last conventional therapy, suggesting that participation in a phase I trial is a reasonable option for these patients.

\footnotetext{
Address for correspondence and requests for reprints: Razelle Kurzrock, M.D., The University of Texas M. D. Anderson Cancer Center, Department of Investigational Cancer Therapeutics, Unit 455, 1515 Holcombe Boulevard, Houston, TX 77030, Phone: 713-794-1226, Fax: 713-794-3249, rkurzroc@ mdanderson.org. Correspondence with John Moroney, M.D.: Wright Patterson Medical Center, Division of Gynecologic Oncology, 4881 Sugar Maple Drive, WPAFB, OH 45419, Phone: 937-673-7318, Fax: 937-257-3012, john.moroney@wpafb.af.mil.

Conflict of interest statement: The authors certify that they have no conflicts of interest to report.

Publisher's Disclaimer: This is a PDF file of an unedited manuscript that has been accepted for publication. As a service to our customers we are providing this early version of the manuscript. The manuscript will undergo copyediting, typesetting, and review of the resulting proof before it is published in its final citable form. Please note that during the production process errors may be discovered which could affect the content, and all legal disclaimers that apply to the journal pertain.
} 


\section{Keywords}

Phase I; Cancer; Gynecologic; Ovarian; Uterine; Cervical; Vulvar; Survival; Response

\section{Introduction}

At initial presentation, patients with gynecologic malignancies vary widely with regard to stage, clinical behavior and prognosis; fortunately, many early stage patients are cured with primary surgical or combination therapies[1-4]. However, patients who are diagnosed with recurrent or progressive disease after primary treatment face a uniformly dismal prognosis, with survival across gynecologic disease sites ranging on average from six months to two years [5-9]. The reason for this, as is with the case with many different solid tumor types, is that there are few effective treatment options for these patients. Most patients undergo a number of toxic, increasingly ineffective palliative therapies before succumbing to their disease[5,8,10].

With increasing awareness of the critical need for more effective and less toxic treatment options for patients with refractory, advanced malignancies, there have been a large number of novel, targeted therapies developed using understanding gained from genetic and molecular profiling of malignancies[11-21]. These are available to patients with advanced gynecologic malignancies through clinical trials. Because of the relatively recent entry into the clinic of such investigational agents, however, many are still in early phase trials, a stage at which there is limited knowledge of efficacy.

Historically, few gynecologic cancer patients are referred for consideration of a Phase I trial. This is likely for a number of reasons, chief among them concerns among oncologists regarding the questionable usefulness of such therapies in patients with advanced cancers[22]. For this reason, we sought to examine the outcome of patients with advanced gynecologic malignancies who were enrolled into phase I trials in the context of a large phase I trials department at a tertiary cancer center.

\section{Patients and Methods}

We reviewed the records of eighty-nine consecutive patients who were seen in the Phase I clinical trials program at M. D. Anderson from March 2006 to April 2008. Phase I trials performed in the department include first-in-human studies, as well as new combinations of experimental and approved drugs. Patients with a gynecologic cancer of any disease site and any histology were included. Patient characteristics, toxicity, response, and survival data were abstracted from electronic medical records. In particular, patient records were reviewed for each patient's prior treatment history, laboratory and clinical findings at the time of initial consultation with the phase I department, protocol treatment rendered, and clinical outcome. All patients evaluated for a phase I trial were referred from the gynecologic oncology department within our institution.

Patients who were enrolled on any trial met the following eligibility criteria. They were at least 18 years old with a metastatic and/or un-resectable gynecologic malignancy for which approved curative therapies were no longer effective. All patients had evidence of measurable disease (according to the Response Evaluation Criteria in Solid Tumors (RECIST)) or evaluable disease [23]. Also, eligible patients had an Eastern Cooperative Oncology Group (ECOG) performance status $\leq$ two and life expectancy of $\geq$ three months. Prior participation in clinical trials and prior biologic (targeted therapies and antibody-based therapies) treatments were not exclusion criteria; however a washout period of at least three weeks preceded the initiation of each phase I therapy. Further eligibility criteria varied according to particular 
studies. All patients completed an informed consent process prior to enrollment onto a trial and all trials were approved by the M. D. Anderson Institutional Review Board (IRB). This study was also conducted in accordance with IRB guidelines.

After initiation of an investigational therapy, patients were evaluated at one to four week intervals. At each visit, a history and physical examination were performed and a metabolic and hematologic panel was conducted. Patients were assessed for the onset of new symptoms, medicine compliance, and toxicities. Toxicities were then judged as not, possibly, probably or definitely related to the study drug. Dose adjustments were made as needed for toxicity. Response to treatment was assessed with imaging studies at six to eight week intervals.

\section{Endpoints and statistical methods}

Descriptive statistics were used to summarize the patients' demographic and clinical characteristics. The Chi-square test was used to examine the association between two categorical variables. The following covariates were analyzed: age, race, ECOG performance status, disease primary (ovarian, uterine, cervix, other), histology, baseline tumor marker levels at referral, history of pelvic radiotherapy (curative intent), number of prior therapies, site(s) of metastases, hydronephrosis, absolute neutrophil count, hemoglobin level, platelet count, albumin, lactate dehydrogenase, calcium, phosphorous, alkaline phosphatase, bilirubin and serum creatinine levels.

Best response was assessed by an M. D. Anderson radiologist starting after two cycles of therapy, and every two cycles thereafter (cycle = three to four weeks, depending on the protocol) using RECIST criteria[23]. These criteria defined a partial response (PR) as a $\geq 30 \%$ decrease in the sum of the longest diameter of target lesions, excluding complete disappearance of disease (CR). Progressive disease was defined as a $\geq 20 \%$ increase in the sum of the longest diameter of target lesions. Stable disease (SD) was defined as any change that did not meet criteria for a PR or progressive disease (PD). For patients treated with more than one therapy, each therapy is described and included in the calculation of response rates. Waterfall plot analysis was used to capture the anti-tumor efficacy of various therapies, as previously described[24]. Responses shown in the waterfall plot were grouped according to standard RECIST criteria.

Survival was measured from date of presentation to the phase I clinical trials program until death from any cause. Patients still alive (overall survival analysis) at last follow-up were censored on that date. Time-to-treatment failure (TTF) was measured from the trial enrollment date to disenrollment or death from any cause, whichever interval was shorter. Patients still on treatment at time of last follow-up were censored on that date. Time on each patient's last therapy (most recent conventional therapy prior to phase I referral) was calculated from the date of first treatment until referral to the Phase I department. When exact dates were not ascertainable, these dates were rounded up to the nearest semi-weekly date. Survival and timeto-treatment failure were plotted using the method of Kaplan and Meier. Toxicities were assessed using the National Cancer Institute Common Terminology Criteria (NCI CTC) for Adverse Events, v. 3.0[25]. A p-value < 0.05 was considered statistically significant. Statistical analyses were carried out using SAS 9.1 (SAS Institute, Cary, NC) and S-Plus, version 7.0 (Insightful Corp., Seattle, WA).

\section{Results}

\section{Patient characteristics}

Of the first 89 patients who were referred for a phase 1 evaluation, 85 (96\%) were enrolled and treated on a trial. Three of the 89 patients developed a deteriorating performance status prior 
to treatment, requiring disenrollment and transition to comfort care; one patient voluntarily withdrew consent prior to treatment. The 85 patients who participated in a phase I clinical trial were included in this analysis. Disease sites were grouped as follows: cervix $(n=17)$, ovary + fallopian tube + peritoneal $(n=43)$, uterus $(n=19)$, vulva $(n=5)$ and gestational trophoblastic neoplasia $(\mathrm{GTN})(\mathrm{n}=1)$. High grade serous $(41 \%)$, squamous $(13 \%)$ and endometrioid $(12 \%)$ were the predominant histologies (Table 1). Patient baseline characteristics at presentation to the phase I clinic are also summarized in Table 1. The median age was fifty-eight years (range, 23 - 78 years). The most common metastatic sites at time of referral were: lymph nodes $(91 \%)$, bulky abdominal metastases (non-nodal metastases $\geq 3 \mathrm{~cm}=70 \%$ ) and liver $(46 \%)$. The median time from diagnosis of cancer to phase I referral was 8.7 years (range 120 days - 12.2 years). The median number of prior total treatments excluding surgery and radiotherapy was six (range 1 to 16). The median number of prior cytotoxic regimens was five (range 1 to 11). Fifty-six patients $(78 \%)$ had one or more medical co-morbidities. The most common co-morbidities were hypertension (35\%), renal insufficiency (creatinine $\geq 1.5,25 \%$ ), thrombosis $(14 \%)$ and hypothyroidism (14\%). Eight patients had a concurrent or preceding malignancy (breast cancer, $\mathrm{n}=5 ;$ renal, $\mathrm{n}=1$; ovarian, $\mathrm{n}=1$; cervix, $\mathrm{n}=1$ ).

\section{Treatment}

The 85 patients treated were enrolled on 48 different phase I trials. 21 (44\%) of these trials were "first-in-human" trials employing targeted inhibitors (18/21), or novel cytotoxics (3/21) as monotherapy. The remaining 27 phase I trials involved novel combinations of targeted agents with cytotoxic chemotherapeutics or novel combinations of targeted agents (two or three drugs). With regard to mechanism, a large number of different types of agents were used. Of the 21 first-in-human trials, six (29\%) involved drugs targeting VEGF signaling. 32 patients $(37 \%)$ were treated in a first-in-human phase I trial. 22 patients $(26 \%)$ were treated with a combination of targeted agents, 22 patients $(26 \%)$ were treated with a combination of targeted agents(s) with cytotoxics, and nine (11\%) were treated with cytotoxic chemotherapy alone.

\section{Response}

Response based on tumor measurements was assessed approximately every two cycles. A waterfall plot showing best response by RECIST for each patient's first and second phase I trial is shown in Figures 1, Panel A and B, respectively. All patients treated are included in the waterfall plot. Of the 85 patients treated on a first phase I trial, however, 21 were not restaged with imaging exams because they discontinued treatment early due to symptoms consistent with early progression $(\mathrm{N}=15)$, social reasons unrelated to drug $(\mathrm{N}=4)$ or toxicity $(\mathrm{N}=2)$. These 21 patients are designated as progressive disease (and included on the plot as such (Fig. 1)) and were considered treatment failure at the time of treatment discontinuation. (All progressive disease was plotted as $+20 \%$ increase.)

Of the 85 patients treated on a first phase I trial, two (2.4\%) achieved a CR, four achieved a PR (4.7\%), $(C R+P R=6(7.1 \%))$, and 18 achieved stable disease $(21.2 \%)$. Stable disease lasted at least six months in eight patients $(9.4 \%)$. Therefore, the total number of patients with a CR, PR or stable disease of at least six months was $14(16.5 \%)$.

Of the eighty-five patients who enrolled on a Phase I trial, twenty-five (29\%) were treated on $\geq$ two protocols and twenty-four of these patients were evaluable for response. The one nonevaluable patient was lost to follow-up after cycle 1 , week 1 , presumably due to side effects of the regimen. Of the twenty-five patients, five (20\%) had a PR and eight (32\%) had stable disease (Figure 1B). Four patients (5\%) were noted to have SD for $\geq 6$ months). Therefore, the total number of patients with a PR or stable disease of at least six months on their $2^{\text {nd }}$ phase I trial was $25 \%$. Of the five patients noted to have a PR as part of their $2^{\text {nd }}$ phase I trial, three were found to have PD and two had SD ( $\leq$ four months) as their best response during their 
$1^{\text {st }}$ phase I trial. Three patients were treated with a 3rd protocol and all were found to have progressive disease at $1^{\text {st }}$ restaging.

Combining response data for all trials, a total of 85 patients underwent 113 trials. In this population, the best responses were two CRs (2.4\%), nine PRs (10.6\%) and 12 (14\%) SD for $\geq 6$ months. Therefore, the total number of patients achieving a CR, PR or SD of at least 6 months was $23(27 \%)$.

\section{Survival}

The median survival time was 7.9 months [95\% CI $6.1-10.1$ ]. The 1-year survival rate was $30 \%$ (95\% CI: $21 \%$ to $44 \%$ ). Overall, 58 patients died.

Log rank survival comparisons by best RECIST responses are shown in Table 2. Patients who achieved a CR/PR or SD had significantly longer overall survival than those patients who had $\mathrm{PD} /$ not evaluable (14.9 vs. 6.0 months; $\mathrm{p}<0.0001$ ). Patients who achieved SD had significantly longer survival than patients who had PD/not evaluable (11 vs. 6 months; $\mathrm{p}=0.004$ ).

Multivariate analysis using a Cox proportional hazards model identified poor ECOG status ( 0 vs. 1 and 0 vs. $2 ; \mathrm{p}=0.05$ and 0.03 , respectively), bone metastases $(\mathrm{p}=0.0007)$, ALT $>56$ ( $=0.008)$, creatinine $>1.5(0.05)$ and the presence of ascites $(\mathrm{p}=0.005)$ as significant prognostic factors for worse overall survival.

\section{Time to treatment failure}

A paired log-rank test was used to compare time to treatment failure (TTF) for different groups. Of the 85 patients enrolled on a $1^{\text {st }}$ phase I trial, comparable data between each patient's treatment immediately prior to phase I referral was available for 76 patients. There was no difference in TTF between patient's prior therapy ( 2.3 months) and their first phase I trial (2.0 months), $\mathrm{p}=0.16$ (Figure 2). Interestingly, there was a trend towards longer TTF for patients' $2^{\text {nd }}$ phase I trial (3.8 months) versus their last conventional therapy prior to phase I referral (2.3 months), though this did not reach significance $(\mathrm{p}=0.09)$ (Figure 3$)$.

\section{Discussion}

Treatment planning for patients with progressive or recurrent gynecologic cancers can be challenging because there is little definitive data regarding optimal therapy in patients who have failed $1^{\text {st }}$ line agents[6-10,26-28]. Unfortunately, response rates and differences in overall survival between different salvage therapies across nearly all gynecologic cancers are uniformly disappointing. Fortunately, insights into aberrant molecular signaling that occurs in malignancy have led to the development of newer targeted as well as cytotoxic agents that are increasingly available through early clinical trials $[9,11,17,18,21]$. While this will hopefully translate to improved outcomes for patients in the future, there has until recently been little published data available for use in evaluating the issue of outcome for patients with gynecologic cancers who enroll on a phase I trial.

In the largest reported series of cancer patients treated on phase I trials ( $\mathrm{N}=460 \mathrm{NCI}$ trials), Horstmann et al. documented a response rate (CR + PR) of 10.5\% among 11,935 participants with diverse malignancies, $54 \%$ of whom received exclusively cytotoxic therapies [29]. This was a seminal paper, especially because of the large size of the population studied, and it has subsequently spurred debate regarding the issue of real vs. "perceived" benefit for phase I trial patients [22,30-33]. However, this report did not address cancer histologies / disease sites treated; therefore extrapolation of response and survival data from this trial to specific solid tumors is problematic, particularly for gynecologic cancers, which in the progressive or recurrent setting, are rarely indolent and are notoriously chemo-refractory. For this reason, we 
sought to examine and describe outcomes for gynecologic cancer patients in a dedicated phase I clinic at a tertiary cancer center.

Historically, meaningful interpretation of response and survival data in cancer trials is difficult because of the extreme heterogeneity inherent in such trials (inclusion of multiple different cancer types as well as differing, but considerable amounts of pre-treatment prior to referral). Some investigators have postulated that a relevant comparison with regard to patient benefit in advanced disease is a paired evaluation of each patients' time to treatment failure on serial therapies [31,32]. The natural history of successive salvage therapies in progressive cancer is that each subsequent therapy has a decreasing likelihood of inducing a response, and on average, the amount of time each patient is on therapy prior to progression decreases [5]. Given these facts, using each patient as his/her own control is a reasonable way in which to explore the question of patient benefit.

Our analysis demonstrates that the time-to-treatment failure for patients entered on a phase I trial was equivalent to that for their prior conventional therapy (Figure 3) $(\mathrm{P}=0.16)$. Interestingly, for 25 patients who enrolled on a second phase I trial, their time-to-treatment failure comparison showed a trend, albeit not statistically significant, toward a longer duration than their previous conventional therapy (median 3.8 vs. 2.4 months; $p=0.09$ ) (Table 3). While the small number of patients in this group precludes definitive assessment of this phenomenon, it is possible that with increasing experience treating gynecologic cancers, patients were assigned to trials that had shown evidence of benefit. An alternative explanation might be that these patients had an unusual natural history that resulted in more prolonged response or stabilization of disease with progressive accumulation of therapeutic regimens, though this seems implausible based on the known clinical course of tumors.

With regard to survival, the median survival of these patients was 7.9 months, and approximately $30 \%$ of patients were alive at one year. Poor performance status, renal or hepatic impairment, bone metastases, and ascites predicted for a worse outcome in multivariate analysis. Patients who achieved a CR, PR or SD fared significantly better than patients found with PD at $1^{\text {st }}$ restaging (median survival $=14.9$ vs. 6.0 months; $\mathrm{p}<0.0001$ ); patients who achieved SD also had significantly longer survival than patients who had PD (11 vs. 6 months; $p=0.004)$. While this is intuitive with regard to CR and PR vs. PD, the fact that patients with SD live statistically longer than with patients with PD suggests that, in patients such as these with advanced, heavily pre-treated disease, prolonged stable disease may be a legitimate measure of benefit. Alternatively, it is possible that prolonged stable disease (as well as CR/ $\mathrm{PR}$ ) in these patients serve as a surrogate for a less aggressive malignant phenotype.

Our data represents the largest examination of gynecologic patients in a phase I clinic to date, with a response rate $(\mathrm{CR}+\mathrm{PR})$ in 85 heavily pre-treated patients (median number prior therapies $=5$ ) of $13 \%$ (when the total of 113 trials that these patients were exposed to are considered). Including patients with stable disease $\geq$ six months, 23 patients (27\%) showed salutary effects.

Gynecologic oncologists are frequently faced with treatment planning dilemmas for patients with chemo-refractory recurrent or progressive cancers. In recent years the development of an increasing number of targeted therapies that are available through early clinical trials has made the option of referral to a phase I clinic a possibility for more patients. Our data would suggest that patients with advanced, heavily-treated disease enrolled on a phase I trial fare at least as well as they did with their prior conventional therapy, and that a small, but not insignificant, subset can attain an objective response or stabilization of disease. 


\section{Acknowledgments}

This work was supported in part by Grant Number RR024148 from the National Center for Research Resources, a component of the NIH Roadmap for Medical Research (http://nihroadmap.nih.gov/clinicalresearch/overview-translational.asp)

\section{References}

1. Lu KH. Management of early-stage endometrial cancer. Semin Oncol 2009;36(2):137-44. [PubMed: 19332248]

2. Kwon YS, et al. Fertility preservation in patients with early epithelial ovarian cancer. J Gynecol Oncol 2009;20(1):44-7. [PubMed: 19471664]

3. Lee CM, et al. Frequency and effect of adjuvant radiation therapy among women with stage I endometrial adenocarcinoma. Jama 2006;295(4):389-97. [PubMed: 16434629]

4. Warwick J, et al. Defining the surgical management of suspected early-stage ovarian cancer by estimating patient numbers through alternative management strategies. Bjog 2009;116(9):1225-41. [PubMed: 19485991]

5. Armstrong DK. Relapsed ovarian cancer: challenges and management strategies for a chronic disease. Oncologist 2002;7:20-8. [PubMed: 12324630]

6. Fleming GF. Systemic chemotherapy for uterine carcinoma: metastatic and adjuvant. J Clin Oncol 2007;25(20):2983-90. [PubMed: 17617530]

7. Martin LP, Schilder RJ. Management of recurrent ovarian carcinoma: current status and future directions. Semin Oncol 2009;36(2):112-25. [PubMed: 19332246]

8. Ray M, Fleming G. Management of advanced-stage and recurrent endometrial cancer. Semin Oncol 2009;36(2):145-54. [PubMed: 19332249]

9. Tewari KS, Monk BJ. Recent achievements and future developments in advanced and recurrent cervical cancer: trials of the gynecologic oncology group. Semin Oncol 2009;36(2):170-80. [PubMed: 19332251]

10. Vermorken JB. Second-line randomized trials in epithelial ovarian cancer. Int J Gynecol Cancer 2008;18:59-66. [PubMed: 18336403]

11. Steelman LS, et al. Akt as a therapeutic target in cancer. Expert Opin Ther Targets 2008;12(9):113965. [PubMed: 18694380]

12. Bansal N, Yendluri V, Wenham RM. The molecular biology of endometrial cancers and the implications for pathogenesis, classification, and targeted therapies. Cancer Control 2009;16(1):813. [PubMed: 19078924]

13. Bast RC Jr, Hennessy B, Mills GB. The biology of ovarian cancer: new opportunities for translation. Nat Rev Cancer 2009;9(6):415-28. [PubMed: 19461667]

14. Catasus L, et al. Concomitant PI3K-AKT and p53 alterations in endometrial carcinomas are associated with poor prognosis. Mod Pathol 2009;22(4):522-9. [PubMed: 19234438]

15. Chaudhry P, Asselin E. Resistance to chemotherapy and hormone therapy in endometrial cancer. Endocr Relat Cancer 2009;16(2):363-80. [PubMed: 19190080]

16. Cho YH, et al. Mutational analysis of KRAS, BRAF, and TP53 genes of ovarian serous carcinomas in Korean women. Yonsei Med J 2009;50(2):266-72. [PubMed: 19430562]

17. Gadducci A, et al. Molecular target therapies in endometrial cancer: from the basic research to the clinic. Gynecol Endocrinol 2008;24(5):239-49. [PubMed: 18569027]

18. Hennessy BT, et al. Exploiting the PI3K/AKT pathway for cancer drug discovery. Nat Rev Drug Discov 2005;4(12):988-1004. [PubMed: 16341064]

19. Honda $\mathrm{H}$, et al. Serial analysis of gene expression reveals differential expression between endometriosis and normal endometrium. Possible roles for AXL and SHC1 in the pathogenesis of endometriosis. Reprod Biol Endocrinol 2008;6:59. [PubMed: 19055724]

20. Ollikainen M, et al. Patterns of PIK3CA alterations in familial colorectal and endometrial carcinoma. Int J Cancer 2007;121(4):915-20. [PubMed: 17471559]

21. Spannuth WA, Sood AK, Coleman RL. Angiogenesis as a strategic target for ovarian cancer therapy. Nat Clin Pract Oncol 2008;5(4):194-204. [PubMed: 18268546] 
22. Miller FG, Joffe S. Benefit in phase 1 oncology trials: therapeutic misconception or reasonable treatment option? Clin Trials 2008;5(6):617-23. [PubMed: 19029210]

23. Eisenhauer EA, et al. New response evaluation criteria in solid tumours: revised RECIST guideline (version 1.1). Eur J Cancer 2009;45(2):228-47. [PubMed: 19097774]

24. Wheler J, et al. Survival of patients in a Phase 1 Clinic: the M. D. Anderson Cancer Center experience. Cancer 2009;115(5):1091-9. [PubMed: 19165805]

25. Trotti A, et al. CTCAE v3.0: development of a comprehensive grading system for the adverse effects of cancer treatment. Semin Radiat Oncol 2003;13(3):176-81. [PubMed: 12903007]

26. Markman M. Unresolved issues in the chemotherapeutic management of gynecologic malignancies. Semin Oncol 2006;33(2 Suppl 6):S33-8. [PubMed: 16716801]

27. Pectasides D, et al. Chemotherapy for recurrent cervical cancer. Cancer Treat Rev 2008;34(7):60313. [PubMed: 18657909]

28. Pignata $S$, et al. Extending the platinum-free interval with a non-platinum therapy in platinumsensitive recurrent ovarian cancer. Results from the SOCRATES Retrospective Study. Oncology 2006;71(5-6):320-6. [PubMed: 17878745]

29. Horstmann E, et al. Risks and benefits of phase 1 oncology trials, 1991 through 2002. N Engl J Med 2005;352(9):895-904. [PubMed: 15745980]

30. Kurzrock R, Benjamin RS. Risks and benefits of phase 1 oncology trials, revisited. N Engl J Med 2005;352(9):930-2. [PubMed: 15745986]

31. Markman M. Further evidence of clinical benefit associated with participation in phase I oncology trials. Br J Cancer 2008;98(6):1021-2. [PubMed: 18349816]

32. Markman M. Therapeutic intent in the conduct of phase I antineoplastic drug trials. Oncology 2009;76 (3):149-50. [PubMed: 19169045]

33. Stewart DJ, Kurzrock R. Cancer: the road to Amiens. J Clin Oncol 2009;27(3):328-33. [PubMed: 19064964] 
panel A

\section{1st phase I trial best RECIST response}

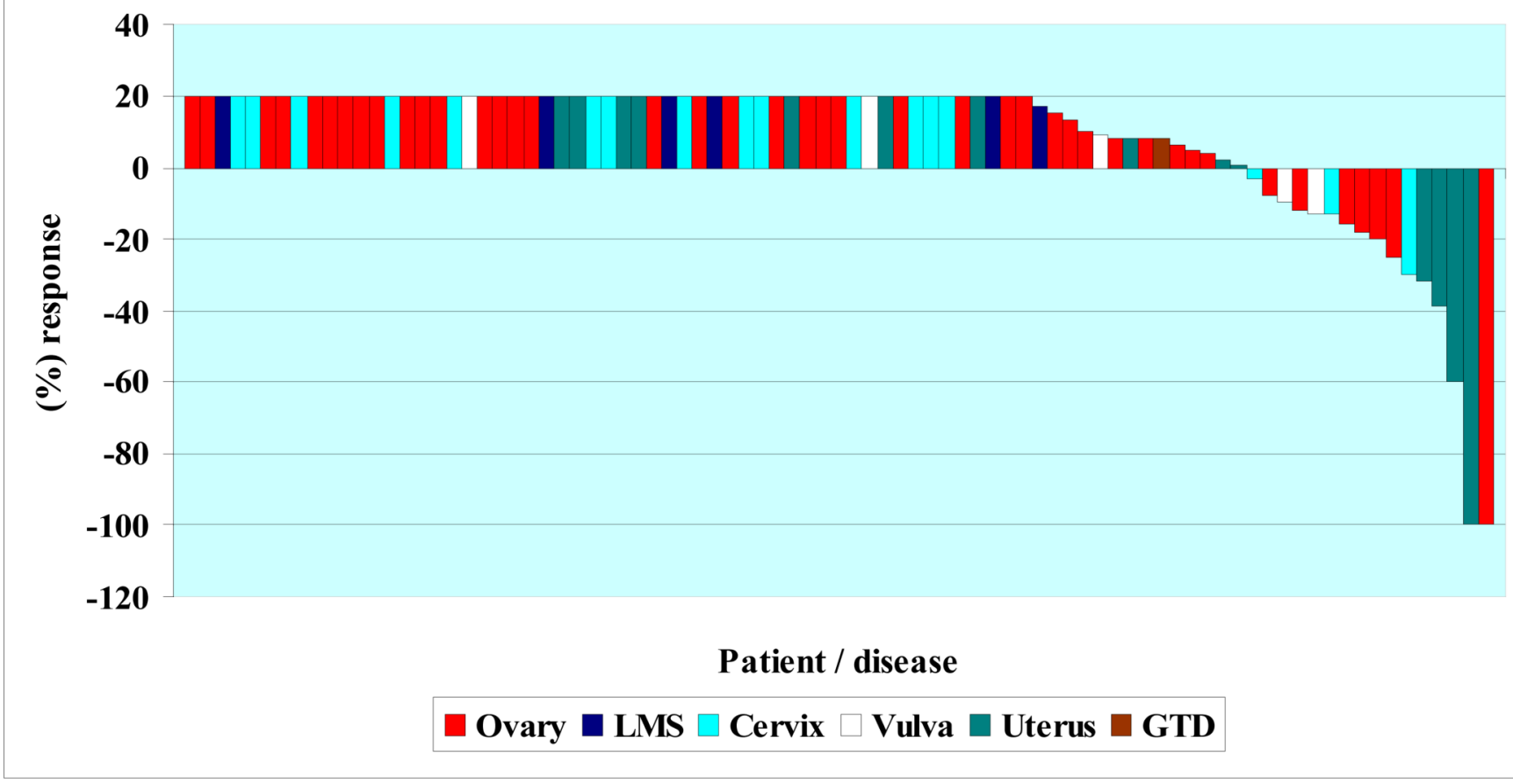

\section{LMS = leiomyosarcoma}

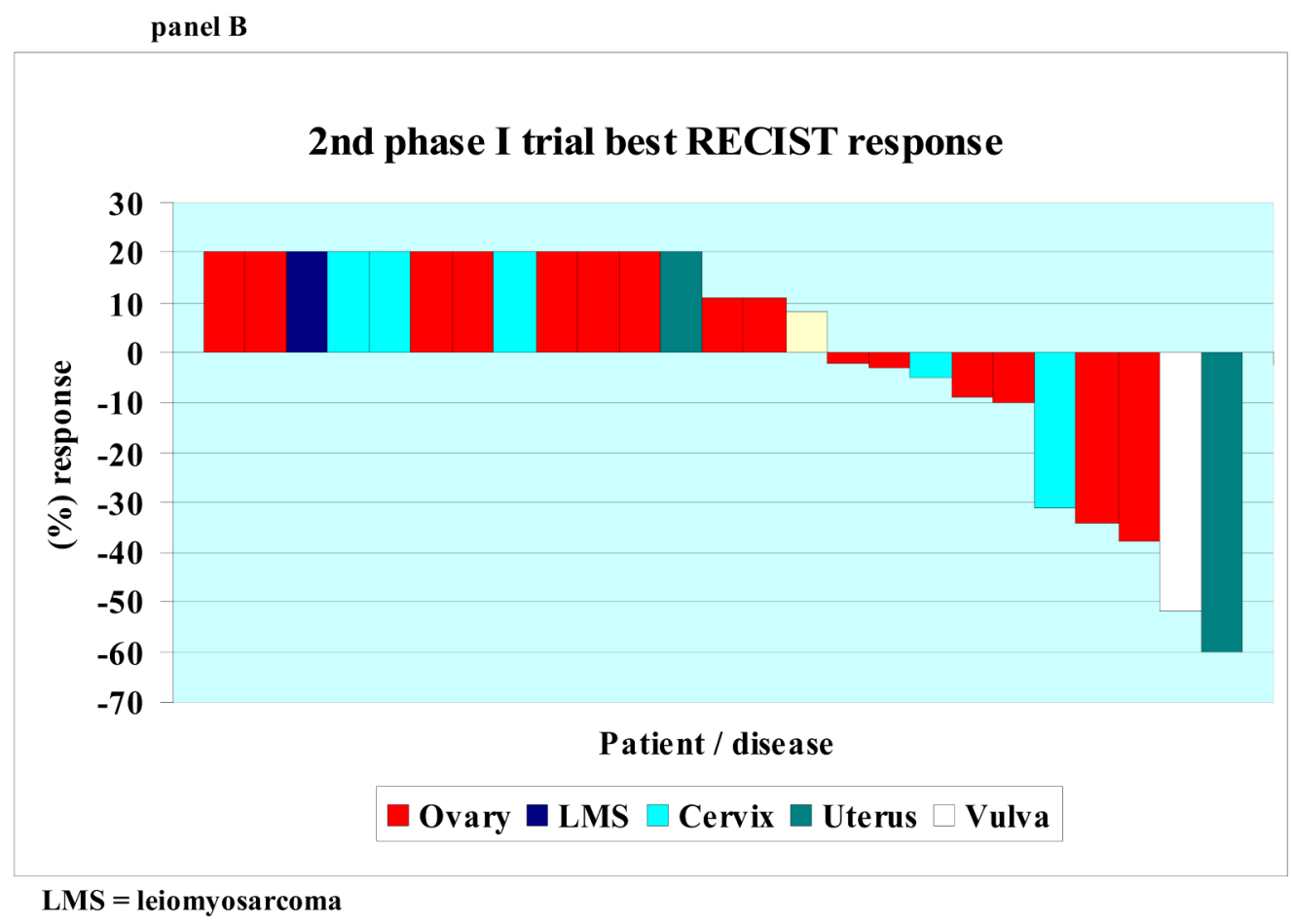


Figure 1.

Panel A: $1^{\text {st }}$ phase I trial best RECIST response Waterfall plot of 85 patients showing best RECIST response to first phase I trial. Patients with progressive disease reflected by increase of at least 20 percent by RECIST, new lesions, or clinical progression are shown as an increase of $20 \%$. Patients who were not evaluable were considered progressive and shown as an increase of $20 \%$.

Panel B: $2^{\text {nd }}$ phase I trial best RECIST response Waterfall plot of 25 patients showing best RECIST response to first phase I trial. Patients with progressive disease reflected by increase of at least 20 percent by RECIST, new lesions, or clinical progression are shown as an increase of $20 \%$. Patients who were not evaluable were considered progressive and shown as an increase of $20 \%$. 


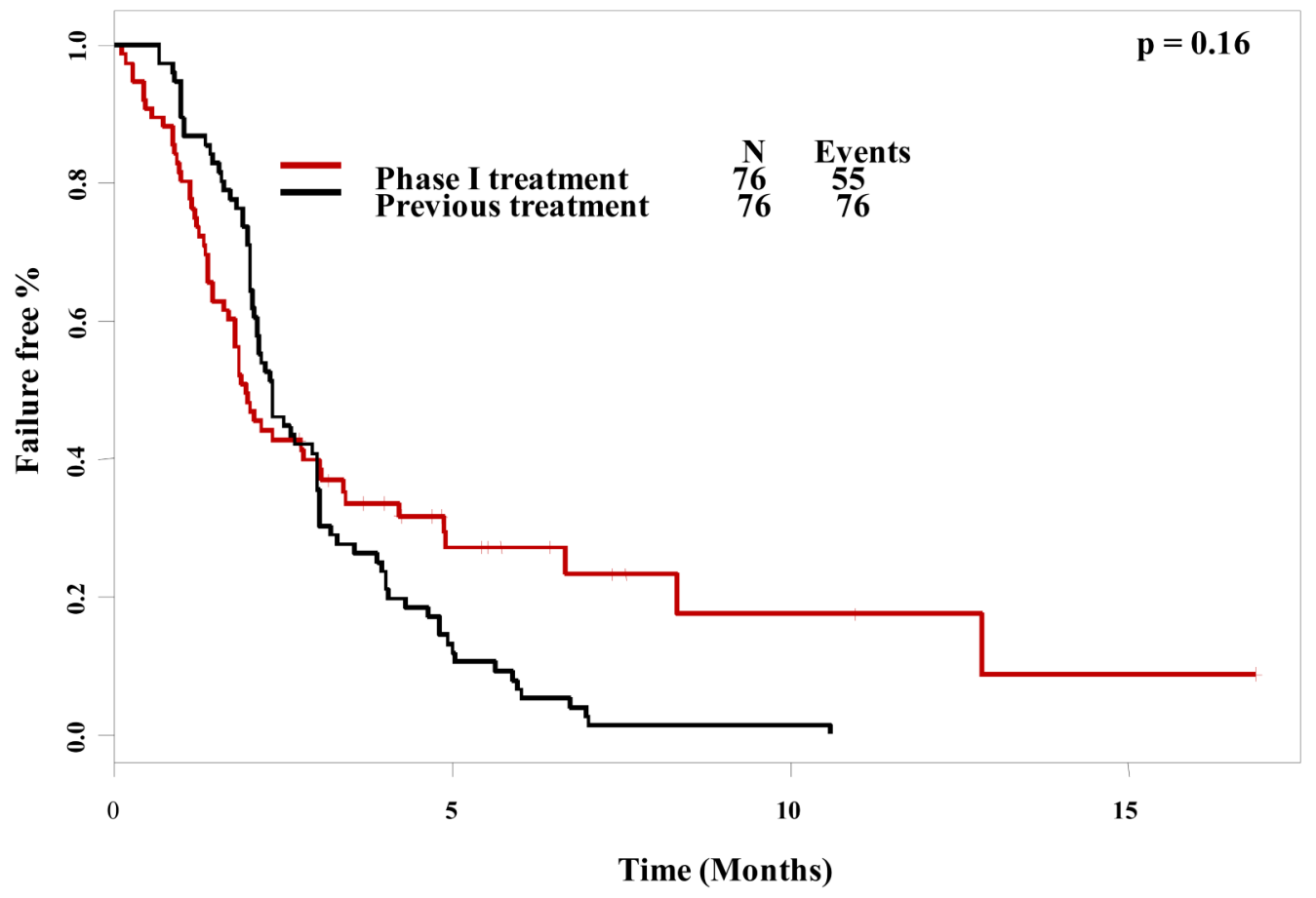

Figure 2. Paired time to treatment failure comparison $\left(1^{\text {st }}\right.$ phase I trial vs. last conventional treatment)

Paired time-to-treatment failure as plotted by Kaplan-Meier for first phase I therapy compared to the last conventional treatment before referral to the phase I clinic. Tic marks represent patients still on treatment and therefore censored at the time shown. Time-to-treatment failure was not significantly different between the first phase I treatment and the last conventional treatment. 


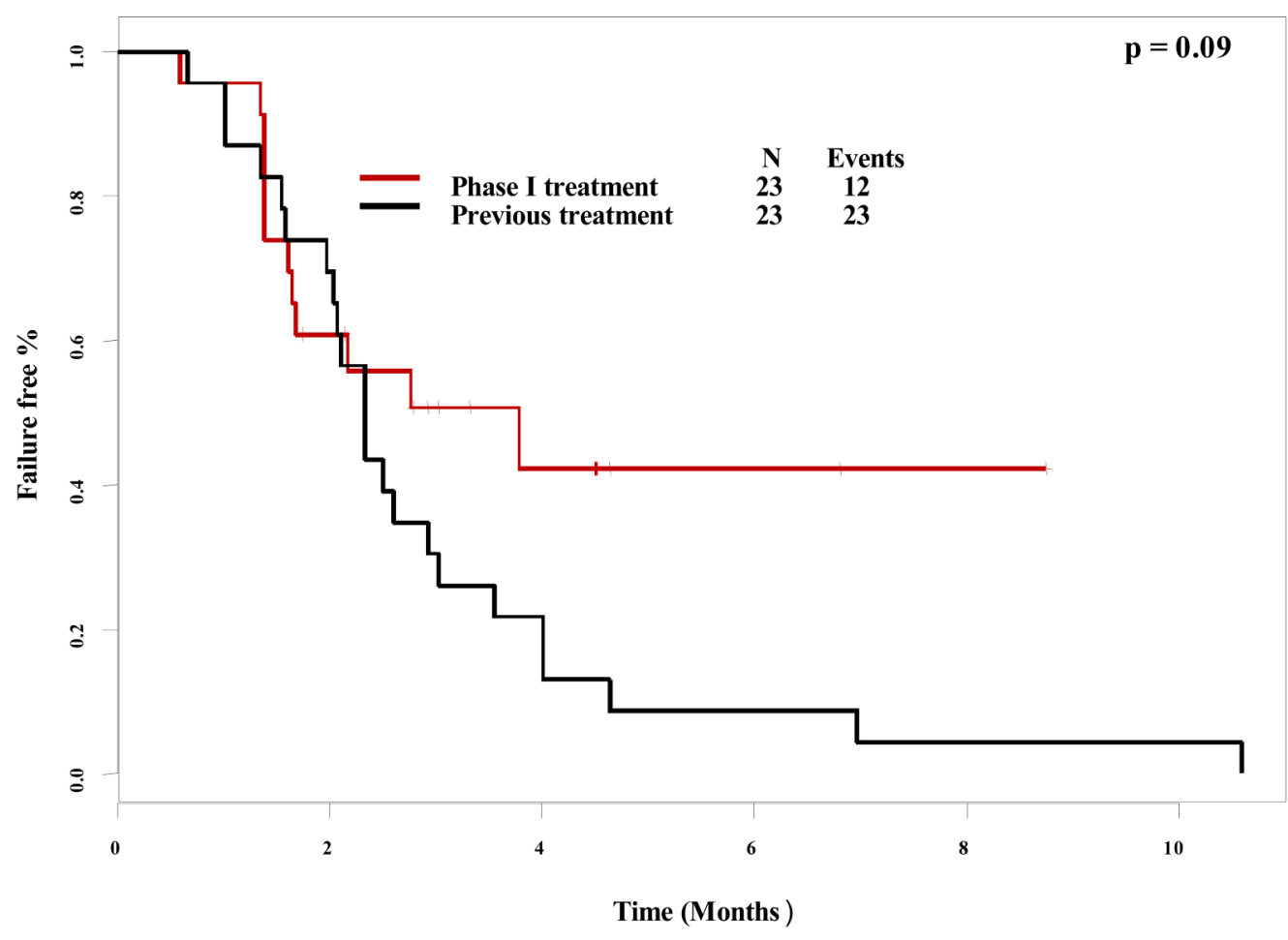

Figure 3. Paired time to treatment failure comparison $\left(2^{\text {nd }}\right.$ phase I trial vs. last conventional treatment)

Paired time-to-treatment failure as plotted by Kaplan-Meier for second phase I therapy compared to the last conventional treatment before referral to the phase I clinic. Tic marks represent patients still on treatment and therefore censored at the time shown. There was a trend, albeit not statistically significant, towards a longer time-to-treatment failure for patients' second phase I trial as compared to their last conventional treatment. 
Table 1

Patient Characteristics

\begin{tabular}{|c|c|}
\hline Characteristic & Number of patients \\
\hline Median age (range) & $58(23-79)$ \\
\hline Median number of prior treatments (range) & $6(1-16)$ \\
\hline Median number of prior cytotoxics (range) & $5(1-11)$ \\
\hline \multicolumn{2}{|l|}{ Cancer diagnosis } \\
\hline Ovary & 43 \\
\hline Uterus & 19 \\
\hline Cervix & 17 \\
\hline Vulva & 5 \\
\hline Gestational Trophoblastic Neoplasia & 1 \\
\hline \multicolumn{2}{|l|}{ Histology } \\
\hline Undifferentiated & 6 \\
\hline High grade serous & 30 \\
\hline Low grade serous & 1 \\
\hline Squamous & 11 \\
\hline Endometrioid & 12 \\
\hline Endometrial stromal sarcoma & 1 \\
\hline Adenocarcinoma & 5 \\
\hline Neuroendocrine & 3 \\
\hline Malignant mixed mullerian & 2 \\
\hline Leiomyosarcoma & 7 \\
\hline Clear cell & 4 \\
\hline Adenosquamous & 2 \\
\hline Placental site trophoblast & 1 \\
\hline
\end{tabular}




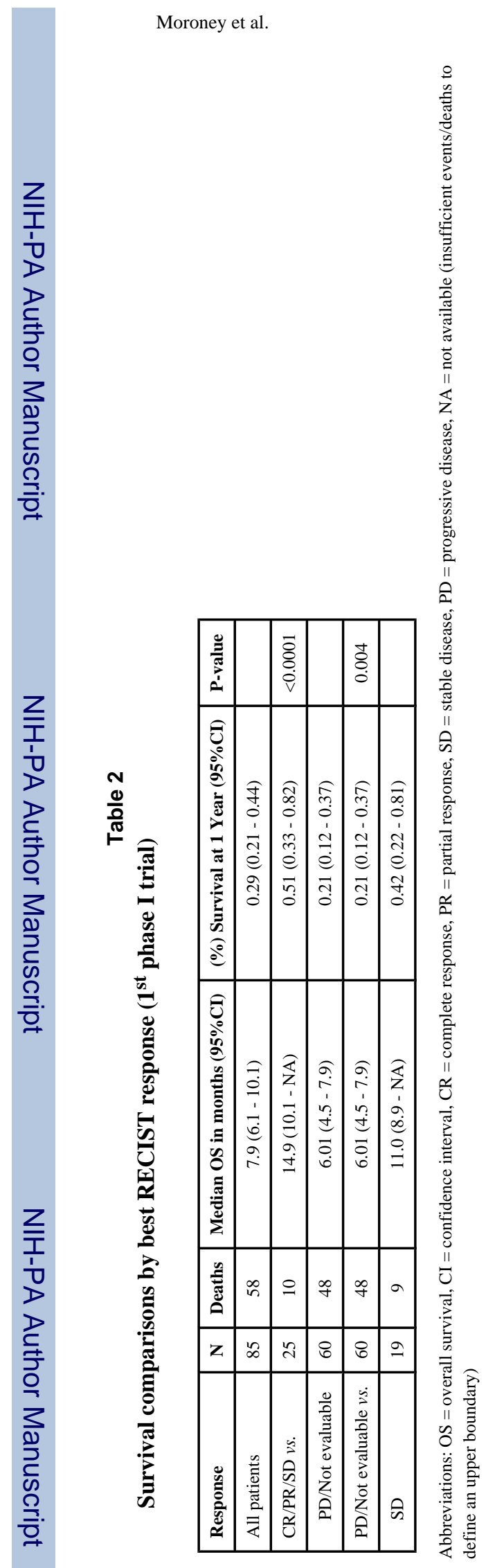

Page 14

Gynecol Oncol. Author manuscript; available in PMC 2011 June 1. 